\title{
Correlations of global sea surface temperatures with the solar wind speed
}

\author{
Limin Zhou ${ }^{1}$, Brian Tinsley ${ }^{2}$, Huimin $\mathrm{Chu}^{1}$, Ziniu Xiao ${ }^{3}$
}

1 Key Laboratory of Geographic Information Science, Ministry of Education, East China Normal University, Shanghai, China, 200062

2 University of Texas at Dallas, Richardson, TX, 75080

3 State Key Laboratory of Numerical Modeling for Atmospheric Sciences and Geophysical Fluid Dynamics, Institute of Atmospheric Physics, Chinese Academy of Sciences, Beijing, China, 100910

\section{Abstract}

A significant correlation between the solar wind speed (SWS) and sea surface temperature (SST) in the region of the North Atlantic Ocean has been found for the Northern Hemisphere winter from 1963 to 2010, based on 3-month seasonal averages. The correlation is dependent on $B_{z}$ (the interplanetary magnetic field component parallel to the Earth's magnetic dipole) as well as the SWS, and somewhat stronger in the stratospheric quasi-biennial oscillation (QBO) west phase than in the east phase. The correlations with the SWS are stronger than those with the F10.7 parameter representing solar UV inputs to the stratosphere. SST responds to changes in tropospheric dynamics via wind stress, and to changes in cloud cover affecting the radiative balance. Suggested mechanisms for the solar influence on SST include 
changes in atmospheric ionization and cloud microphysics affecting cloud cover, storm invigoration, and tropospheric dynamics. Such changes modify upward wave propagation to the stratosphere, affecting the dynamics of the polar vortex. Also, direct solar inputs, including energetic particles and solar UV, produce stratospheric dynamical changes. Downward propagation of stratospheric dynamical changes eventually further perturbs tropospheric dynamics and SST.

Atmospheres 3305

Key words: solar wind speed, sea surface temperature, clouds, global electric circuit 


\section{Introduction}

\subsection{Linkages and timescales}

There is much evidence of significant tropospheric responses to space weather and space climate in the form of variations of galactic cosmic rays (GCR) (Tinsley and Deen 1991; Svensmark and Friis-Christensen 1997; Artamonova and Veretenenko 2011), solar energetic particle events (SEP) (Veretenenko and Thejll 2004; Mironova et al. 2012a; Mironova and Usoskin 2013, 2014), interplanetary magnetic field (IMF) (Tinsley and Heelis 1993). A mechanism has been proposed by which the global electric circuit is influenced immediately by the above inputs from the solar wind, with the downward current density $\left(J_{z}\right)$ affecting meteorology via changes in cloud microphysics (Markson and Muir 1980; Tinsley 1996, 2008, 2012).

A relation between energetic electron precipitation (EEP) with the energy of few hundred $\mathrm{keV}$ and northern polar winter climate variations has been inferred from data analyses by Seppälä et al. (2009) and Andersson et al. (2014), and climate model simulations such as by Rozanov et al. (2005) and Baumgaertner et al. (2011) support an explanation in terms of stratospheric ozone chemistry affecting the atmospheric radiation balance (Rozanov et al. 2012, Seppälä and Clilverd 2014). Analysis of observations by Maliniemi et al. (2013) has shown correlations of the North Atlantic Oscillation (NAO) index and surface temperature with EEP (30-100 keV and 100-300 $\mathrm{keV}$ ) that are significantly stronger during the easterly quasi-biennial oscillation (QBO) phase than in the westerly phase.

Responses to changes in total solar irradiance and solar UV irradiance have been 
inferred by many authors, with more than a thousand reports over the past two centuries (Hoyt and Schatten, 1997), with recent examples including Haigh (1996); Matthes et al. (2006); Gray et al. (2010); and Ermolli et al. (2013). For the solar UV input, which is absorbed in the stratosphere, as well as the EEP input, the connection to tropospheric dynamics is by slow downward propagation on a timescale of weeks to a month or so, involving planetary wave propagation and synoptic-scale Rossby wave breaking. These analyses treat data in three-month and inter-annual and decadal averages, in which there is enough time for downward propagation from the stratosphere to affect the tropospheric dynamics (Baldwin and Dunkerton, 1999; Lu et al. 2013).

Responses on the day-to-day timescale have been extensively reviewed by Tinsley (2008) and Lam and Tinsley (this issue). These responses are consistent with the global circuit-cloud microphysics mechanism and tend to occur when changes in ionospheric potential or atmospheric conductivity occur at locations where clouds or atmospheric instabilities, such as the polar front in winter, are present. For this timescale there is little ambiguity as to which responses are due to which of several solar wind inputs. In contrast with such day-to-day responses, in this paper we are concerned primarily with responses on the inter-annual and decadal timescales, for which so far there has been no reliable way to infer the responsible solar input.

\subsection{Responses in the North Atlantic}

The North Atlantic region seems to be favored for many of the observed responses, as found in the correlation of atmospheric geopotential height $(\mathrm{GPH})$ with solar wind 
geo-effective electric field (GEF) (Boberg and Lundstedt 2003); of GPH with solar wind speed (SWS) (Zhou et al. 2014); and of surface air temperature (SAT) with energetic electron precipitation (EEP) and the geomagnetic activity that accompanies it (Seppälä et al. 2009; Baumgaertner et al. 2011; Maliniemi et al. 2013). The NAO is a regional measure of atmospheric response, and is a mode of variation in atmospheric parameters that is largely coherent from Florida to Greenland and from northwestern Africa to Europe. It is defined by the normalized pressure differences between a low latitude station (e. g., Lisbon) and a high latitude station (e. g., Stykkisholmur, Iceland). The causes of its variability, especially on decadal and longer timescales, are not completely understood, and there seems to be a role for ocean circulation changes and external forcing, with even a suggestion that the "observed inter-annual and longer time scale NAO fluctuations could entirely be a remnant of the energetic weekly variability" (Hurrell et al. 2003). A pattern of SST changes associated with the atmospheric NAO is described by Visbeck et al. (2001).

Large scale dynamical responses to the 11-year cycle of solar activity in the north Atlantic region have been found, for example, by Kodera (2002); Thejll et al. (2003); and Huth et al. (2006, 2009). The strongest North Atlantic responses to the solar wind inputs have been found in the extended northern winter months (November-March) when atmospheric dynamics are strongest and the energy conversions (including cloud processes) are thought to be more easily perturbed by external energy and ionization inputs.

A strong association between stratospheric dynamics and seasonal changes in SST 
has been found with a coupled atmosphere-ocean model by Reichler et al. (2012), through a dynamic linkage that propagates from the stratosphere to the troposphere, with wind stress affecting ocean dynamics and sea surface temperature. The model had no external forcing, and the authors state that it is most likely that the stratospheric energy that propagates down is related to initial stochastic forcing from the troposphere. This raises the possibility that upward wave propagation from day-to-day tropospheric responses to the solar wind cause stratospheric dynamical changes that feed back into tropospheric SST responses on the seasonal and longer timescales.

In this context it is of interest that the study of Thejll et al. (2003) found significant positive correlations between winter (DJFM) sea level pressure and the geomagnetic Ap index (which is a measure of short term solar wind variability) in the North Atlantic from 1973-2001. Earlier than 1973 the pressure/Ap correlations were weaker, and in 1949-1950 were weakly negative. They note that prior to about 1970 the polar vortex was also weaker, and that downward coupling in general is weaker with a weaker vortex. However, for correlations between stratospheric geopotential height and Ap, a significant connection was found by Thejll et al (2003) both before and after 1970. Upward coupling is not found to be affected by the vortex strength (Perlwitz and Graf (2001). Graf and Walter (2005) noted that the polar vortex controls the downward coupling not only to the atmosphere but also to the ocean, as characterized by the NAO.

All this is consistent with a scenario in which day-to-day tropospheric responses to 
solar wind inputs are propagating upwards to the stratosphere and affecting the vortex strength, with downward coupling on a timescale of weeks to months affecting the large scale circulation.

A response of surface pressure in the Arctic and Antarctic to changes in ionospheric potential on a timescale of about a week (the Mansurov effect) has recently been shown to be part of a geopotential height anomaly extending over the whole of both high latitude regions and out into mid-latitudes (Lam et al. 2013), which propagates upwards in altitude (Lam et al. 2014) through the troposphere. It is statistically significant within the troposphere, rather than above it. The changes in ionospheric potential were deduced from radar and spacecraft measurements, and are driven by the product of SWS and the $B_{y}$ component of the solar wind magnetic field. The only candidate mechanism consistent with the electrical input involves $J_{z}$ and cloud microphysics, which is also a candidate for the other day-to-day responses to space weather noted above. Both the IMF $B_{y}$ component and SWS vary on the 11-year solar cycle.

That relativistic electron (2-15 Mev) precipitation (herein REP) could play an important role in coupling solar wind and magnetospheric variability as far down as the middle atmosphere was found by Baker et al. (1987, 1993). The connections between REP and SWS have been demonstrated by Li et al. (2001a, b) and Reeves et al. (2011). The REP can penetrate down to the upper stratosphere levels in the sub-auroral zone (magnetic latitude of $45^{\circ}-65^{\circ}$ ), and Bremsstrahlung radiation is produced at the same time, which can impact stratospheric ozone chemistry as for 
EEP. In addition, the ionization produced by the REP and the Bremsstrahlung can alter the electric conductivity down to lower stratosphere levels, so that the stratospheric electrical column resistance is changed, and also $J_{z}$. The electrical link through $J_{z}$ provides immediate coupling between the ionosphere and the troposphere. On the day-to-day timescale a response of the NAO index to REP has been found (Zhou et al. 2014). However, the strength of the response in terms of atmospheric vorticity was found to depend on the amount of volcanic sulphate aerosol in the middle atmosphere (Kirkland and Tinsley 1996; Kniveton and Tinsley 2004; Mironova et al. 2012b). Because of its dependence on irregular episodes of volcanic aerosol the REP/electrical influence on the NAO is not a good candidate for the decadal SST responses.

In this paper, we analyze data on the effects of SWS, GEF, and the QBO on SST, both globally and with respect to season. We use a large data set from 1963 to 2011, and look at effects in high and low speed ranges of SWS.

\section{Data analysis and methods}

The data sources whose description follows all have URLs specified in the Acknowledgements section. The interplanetary magnetic field (IMF) and SWS monthly data are from the OMNI time series (King and Papitashvili, 2005) from 1963 to 2011. The SWS data are intermittent from 1964 to 1994, continuous from 1995 to 2011, and the data gap has small influence on the monthly data (Finch and Lockwood 2007). The solar wind geo-effective electric field (GEF) is defined by $B_{s} V$, where $V$ is 
the solar wind speed and $B_{s}$ is the southward component of the interplanetary magnetic field, which is negative and equal to $B_{z}$ when $B_{z}<0$ and equal to 0 when $B_{z}$ $\geq 0$, where $B_{z}$ is the interplanetary magnetic field component parallel to the earth's magnetic dipole (Boberg and Lundstedt 2002). A value of GEF for each month is determined by removing from that month all days when for that day the average $B_{z} \geq$ 0 and then averaging $B_{z}$ for the remaining days and multiplying by the average SWS for that month.

The $10.7 \mathrm{~cm}$ solar flux monthly data is obtained from NOAA. When we use the time series of $10.7 \mathrm{~cm}$ solar flux, solar wind electric field and SWS, all of the data is standardized by Z-Score

$$
\text { Z-Score }=(\mathrm{x}-\mu) / \sigma
$$

$\mathrm{x}$ is the specific data, $\mu$ is the average, and $\sigma$ is the standard deviation.

The sea surface temperature is obtained from Hadley Centre Sea Ice and Sea Surface Temperature data set provided by The Met Office Hadley Centre UK, (Rayner et al., 2003). QBO and NAO indices are obtained from NOAA Climate Prediction Center data from 1963 to 2010 . The QBO index is the zonally averaged wind speed measured at $30 \mathrm{hPa}$ above the equator.

We use the wavelet power spectrum analysis to obtain the distribution of periods of the SWS, solar wind geo-effective electric field, $10.7 \mathrm{~cm}$ solar flux and SST. The wavelet analysis follows the method of Torrence and Compo (1998) obtained online, and the base function of Morlet was involved. The correlation coefficients between space parameters and SST are Pearson coefficients, and are depicted by the intensity 
of colors in the following correlation maps with $1^{\circ} \times 1^{\circ}$ longitude-latitude resolution. The data was analyzed as three-month seasonal averages, e.g., for DJF for 1963 the average is over December 1963 and January-February 1964. From the seasonal averages for each year the statistical significance of each grid element was calculated by the Monte Carlo test, depended on 100,000 random time series, and contours of $95 \%$ significance in the following figures are denoted by dashed lines, with contours of $99 \%$ significance by solid lines. The statistical significance was calculated assuming a persistence of two years in the SST, i.e., treating the 48 years as 24 independent samples, estimated from the year-to-year fluctuations in the NAO index illustrated in the next section.

\section{Results}

\subsection{Overview of long term series character of solar activity and North Atlantic}

\section{Oscillation}

Figure 1(a) shows time series of monthly values, in data from 1963 to 2010, of the solar wind speed (the dotted line), the solar wind electric field (the solid line), the $10.7 \mathrm{~cm}$ solar flux (the dashed line), and Figure 1(b) shows the North Atlantic Oscillation (NAO) index (the solid line); while Figure 1(c) shows their power spectra. The results show the strong decadal solar cycle in the three parameters of solar activity. The reason that the GEF time series strongly resembles the F10.7 time series is that it is dominated by $B_{z}$ changes rather than SWS changes, and the largest values of $B_{z}$ occur near the sunspot and F10.7 maxima. The power spectrum of the NAO 
index shows peaks near two and four years, but periods of 8 years and longer are not significant since only 43 years of data are included in the analysis. A much longer time series $(1825$ - 2000) of the NAO index was used in a wavelet analysis by Boberg and Lundstedt (2002) and shows peaks in power density at 8 and 11 years. The decadal cycle of the SWS has a 39 months delay compared with that of the $10.7 \mathrm{~cm}$ solar flux.

\subsection{Correlation coefficient between solar wind speed and sea surface temperature}

In order to study the effect of the SWS on SST, we computed the correlation coefficient between SWS and SST in the four seasons with $1^{\circ} \times 1^{\circ}$ resolution (Figure 2). The data of those parameters are seasonally averaged, with the correlation between SWS and SST being most significant for the northern hemisphere in its winter, DJF, Fig. 2(d). There is a significant negative correlation $(R=-0.52$, over $99 \%$ confidence $)$ between SST and SWS at low latitudes in the North Atlantic ocean, ranging from latitude $5^{\circ} \mathrm{N}$ to $20^{\circ} \mathrm{N}$, and another south of Greenland $(\mathrm{R}=-0.48$, over $99 \%$ confidence) near $58^{\circ} \mathrm{N}, 38^{\circ} \mathrm{W}$. The pattern in the north Atlantic region is similar to the spatial pattern of the NAO (Visbeck et al., 2001).

Some scattered areas $\left(\sim 30^{\circ} \mathrm{S}, \sim 100^{\circ} \mathrm{W}\right)$ east of Easter Island also show negative correlations $(\mathrm{R}=-0.48$, over $99 \%$ confidence). A small region with significant positive correlation area appears in the western Atlantic ocean at mid latitudes near $38^{\circ} \mathrm{N}, 69^{\circ} \mathrm{W}(\mathrm{R}=0.43$, over $95 \%$ confidence). There are also small regions of negative correlation in the south Pacific Ocean. For the other seasons, MAM Fig, 2(a), 
JJA Fig. 2(b), and SON Fig 2(c), similar, but increasingly weaker correlations appear in the progression of seasons following northern winter.

Figure 3 shows the regression of SST on SWS for locations at the peaks of the negative correlations in the north Atlantic at high latitudes (3a) and at low latitudes (3b). The negative slope of the regression approximates $-1^{\circ} \mathrm{C}$ per $100 \mathrm{~km} / \mathrm{s}$ at both sites, with the large scatter precluding a more precise value.

Figure 4 shows spatial distribution of the correlation between solar wind geo-effective electric field (GEF) and SST in panel (a), and $10.7 \mathrm{~cm}$ solar flux and SST in panel (b) in DJF from 1963 to 2010. The GEF correlates with SST with 95\% confidence in a limited area of the north Pacific ocean (around $45^{\circ} \mathrm{N}, 145^{\circ} \mathrm{W}$ ), and near Greenland and Iceland and at mid latitudes to the east of the United States. The $10.7 \mathrm{~cm}$ flux shows a positive correlation with SST with $95 \%$ confidence mainly in the North Pacific Ocean with the center at $45^{\circ} \mathrm{N}, 165^{\circ} \mathrm{E}$ and south Atlantic Ocean with the center at $38^{\circ} \mathrm{S}, 40^{\circ} \mathrm{W}(\mathrm{R}=0.36)$.

Moreover, it is found that the correlation coefficient between SWS and SST in winter is non-linear and depends on the mean speed of solar wind (Figure 5). The SWS data is divided into a high speed phase and low speed phase referred to its long term average $(442.28 \mathrm{~km} / \mathrm{s})$ of 1963-2010 in DJF. Figure 5(a) shows the response of the SST to the SWS in the high speed phase, with a strong positive response $(R=0.59$, with $99 \%$ confidence) in the north Atlantic near $35^{\circ} \mathrm{N}, 58^{\circ} \mathrm{W}$, and a weaker positive response $(95 \%)$ confidence in the Gulf of Alaska $(\mathrm{R}=0.42)$. In the SWS low phase, Fig. 5(b), the areas with significant correlation in the north Atlantic region are larger 
than for the high speed phase, and similar to those of Fig. 2(d), for both phases combined. These areas are the North Atlantic Ocean at low latitudes, to the south of Greenland, and near Easter Island.

Figure 6 shows the maps of the correlation coefficient for DJF between SWS and SST with different $B_{z}$ phases, where the phases are defined by whether the average $B_{z}$ is negative, Fig. 6(a), or positive, Fig. 6(b). For average $B_{z}$ negative the areas with correlations are similar to those of Fig. 2(d); negative correlations southeast of Greenland ( $R=0.52$, over $99 \%$ confidence), in the Atlantic Ocean at low latitudes ( $R$ $=0.46$, over $99 \%$ confidence); in the western Pacific Ocean (center at $28^{\circ} \mathrm{N}, 136^{\circ} \mathrm{E}$ ) and in the center of Indian Ocean (center at $15^{\circ} \mathrm{S}, 70^{\circ} \mathrm{E}$ ). In the positive $B_{z}$ phase, Fig. 6(b), the significant correlation in North Atlantic region disappears but the low latitude correlation in the north Atlantic persists. In the southeast of South America there appear scattered areas with positive correlations and again in the center of the Indian Ocean a small area shows a negative correlation.

Figure 7 shows the results of the correlation coefficient between SWS and SST during different QBO phases. The DJF's QBO phase is defined from the dominant phase of three winter months; if two months have easterly QBO phase, then we define this winter as easterly QBO phase. In the easterly QBO phase, Fig. 7(a), there is a significant negative correlation $(\mathrm{R}=-0.53$, over $99 \%$ confidence $)$ between SST and SWS in the low-latitude North Atlantic on the eastern side, around $28^{\circ} \mathrm{N}, 10^{\circ} \mathrm{W}$; also to the south of Greenland $\left(\mathrm{R}=-0.45\right.$, over $95 \%$ confidence) around $58^{\circ} \mathrm{N}$, $38^{\circ} \mathrm{W}$ and in the south east Pacific ocean $(\mathrm{R}=0.54$, over $99 \%$ confidence $)$ around 
$42^{\circ} \mathrm{S}, 120^{\circ} \mathrm{E}$. Positive correlations can be found north of Hawaii $\left(32^{\circ} \mathrm{N}, 150^{\circ} \mathrm{W}\right)$; in the eastern mid-latitude north Atlantic (near $32^{\circ} \mathrm{N}, 65^{\circ} \mathrm{W}$ ), and to the southeast of South America $\left(55^{\circ} \mathrm{S}, 58^{\circ} \mathrm{E}\right)$. In the westerly QBO phase, Fig. 7(b), the region of significant negative correlation in the low-latitude north Atlantic is now on the western side, ranging from 35 to $60^{\circ} \mathrm{W}$ ( $\mathrm{R}=0.49$, over $99 \%$ confidence). Negative correlation also can be found, as before, to the south of Greenland $\left(30^{\circ} \mathrm{N}, 60^{\circ} \mathrm{W}\right)$; south of Easter Island $\left(43^{\circ} \mathrm{S}, 110^{\circ} \mathrm{W}\right)$; with now a small region northwest of Hawaii $\left(8^{\circ} \mathrm{S}, 170^{\circ} \mathrm{W}\right)$. Small regions with positive correlation can be found south and south east of South America ( $\mathrm{R}=0.43$, over $99 \%$ confidence $)$.

\section{Discussion}

The spatial pattern of correlation of SST with SWS that is seen in Fig. 2(d) in the northern hemisphere is strongest in the northern winter (DJF) and declines in the following nine months, suggesting that the temperature change is due to an atmospheric input in winter and possibly also in early spring. Possible inputs in response to changes in the SWS include changes in atmospheric dynamics affecting wind stress or in cloud cover changes affecting radiative coupling. The NAO is an atmospheric oscillation that can be excited by inputs confined to only a part of its spatial extent. Thus inputs could be occurring at high magnetic latitudes only, such as due to magnetosphere-ionosphere coupling or energetic particle precipitation at sub-auroral latitudes, and not necessarily at the lower latitudes where significant responses are also found. 
The comparison of the DJF correlation map in Fig. 4(a) with that in Fig. 2(d) indicates a somewhat different and weaker response of SST to the GEF than to the SWS. The spatial distribution in Fig. 4(a) is similar to that of SAT as shown by Boberg and Lundstedt (2003, their Fig. 1f), but their use of annual rather than winter averages may account for their region of maximum correlation being in a different area of the north Atlantic than in Fig. 2(d).

The spatial distribution of the SST response to the F10.7 flux is similar to that for the response to the GEF, and again is a weaker response than that to the SWS in Fig. 2(d).

The differing response to the SWS when it is in a range above the long-term average, Fig. 5(a), from that when it is below the long-term average, Fig. 5(b), suggests that the SST response is non-linear with SWS. A non-linearity of a measure of winter storm vigor, known as the Vorticity Area Index (VAI), to the REP, which is dependent on the SWS, was found by Mironova et al. (2012b). However, as noted, the mechanism involving REP is dependent on the presence of stratospheric volcanic aerosols, which is independent of the 11-year solar cycle, so in this case the non-linearity cannot be due to the subset of electrical mechanisms involving the REP input.

In Figure 6(a) we see a stronger SST response to the SWS when $B_{z}$ is negative than in 6(b) when $B_{z}$ is positive, in the high geomagnetic latitude region near Iceland and southern Greenland. This is a different result from that in Fig. 4(a) where the daily average $B_{s}$ is used for the GEF response. Here (Fig. 6) the correlation is with the 
SWS alone, and not its product with $B_{z}$, and the stratification is by the average value of $B_{z}$ over each winter. With negative $B_{z}$ more solar wind energy enters the magnetosphere-ionosphere system than with positive $B_{z}$, increasing energetic particle precipitation and ionospheric electric fields associated with magnetic storms. Increased response with negative $B_{z}$ is not consistent with UV or total irradiance forcing.

The stratification by QBO east, Fig. 7(a), and QBO west, Fig. 7(b), shows a stronger coupling when the average QBO over a winter is in the west phase than when it is the east phase. This is the opposite result to that of Maliniemi et al. (2013) who, however, excluded winters that had strong sudden stratospheric warming before separating the data by QBO phases, and analyzed surface air temperature over regions that included continents as well as oceans. In general, the QBO has a role in the dynamical coupling between the troposphere and the stratosphere, and can strongly affect the stability of the stratospheric vortex in winter (Labitzke, 1987; Labitzke and van Loon, 1988). However, in view of the results of Reichler et al. (2012) mentioned earlier, the effect of the QBO does not allow one to discriminate whether a solar wind input to the atmosphere affecting stratospheric ozone is slowly propagating down to affect the troposphere, or whether a prompt electrical response of clouds and storm dynamics is propagating up to affect the stratosphere, to be followed by downward propagation. 


\section{Conclusions}

Responses of sea-surface temperature to solar wind speed on the seasonal timescale have been found, and in the North Atlantic region in winter they resemble the North Atlantic Oscillation. At the locations of the peak (negative) response in the North Atlantic the SST decreases by approximately $1^{\circ} \mathrm{C}$ for $100 \mathrm{~km} \mathrm{~s}^{-1}$ increase in solar wind speed. In the southern hemisphere regions of significant positive and negative correlations with SWS are smaller and less well organized. The analysis is made for 1963-2010 data, and the effect strongest in winter, and greater when the solar wind magnetic field is southward, ensuring greater energy input into the ionosphere-atmosphere system. The response is somewhat stronger in the west phase of the QBO than in the east phase, and stronger than when F10.7 is used for the correlations. These seasonal responses are consistent with cumulative effects of a prompt electrical mechanism affecting cloud microphysics microphysics, as found elsewhere. However, on the seasonal timescale there is ambiguity as to which of several inputs to the stratosphere or troposphere is driving the responses. It is even possible that upward propagating waves, resulting from short term responses to the solar wind, are changing the strengths of the northern and southern polar vortices, which on longer timescales cause downward propagating dynamical disturbances that affect the NAO and the southern oceans.

\section{Acknowledgements}


This work has been partially funded by Grant 2012CB957804 of the National Key Research Science Programmes of China; by NSFC Grant (41271054); and the US National Science Foundation Grant AGS 0836171. The data sources are the open web sites. The interplanetary magnetic field (IMF) and SWS monthly data are from the OMNI time series (http://omniweb.gsfc.nasa.gov/ow.html). The $10.7 \mathrm{~cm}$ solar flux monthly data is obtained from NOAA (http://www.ngdc.noaa.gov/nndc/struts). The sea surface temperature data set is from The Met Office Hadley Center (UK) (http://www.metoffice.gov.uk/hadobs/hadisst/data/download.html). QBO and NAO indices are obtained from NOAA Climate Prediction Center data (http://www.cpc.ncep.noaa.gov/data/indices/qbo.u30.index and http://www.cpc.ncep.noaa.gov/products/precip/CWlink/pna/nao.shtml). Wavelet software was provided by C. Torrence and G. Compo at http://atoc.colorado.edu/research/wavelets/.

\section{References}

Andersson, M. E., Verronen, P. T., Roger, C. J., Clilverd, M. A., and Seppälä A., 2014. Missing driver in the Sun-Earth connection from energetic electron precipitation impacts on mesospheric ozone. Nature Comms, doi:10.1038/ncomms6197.

Artamonova, I., and Veretenenko, S., 2011. Galactic cosmic ray variation influence on baric systems dynamics at middle latitudes. J. Atmos. Solar. Terr. Phys., 73, 366-370.

Baker, D. N., Blake, J. B., Gorney, D. J., Higbie, P. R., Klebesadel, R. W.and King, 
J. H., 1987. Highly relativistic magnetospheric electrons: A role in coupling to the middle atmosphere. Geophys. Res. Lett., 14, 1027-30.

Baker, D. N., Goldberg, R. A., Herrero, F. A., Blake, J. B. and Callis, L. B., 1993. Satellite and rocket studies of relativistic electrons and their influence on the middle atmosphere. J. Atmos. Terr. Phys., 55, 1619-28.

Baldwin, M. P., and Dunkerton, T. J., 1999. Downward propagation of the Arctic Oscillation from the stratosphere to the troposphere, J. Geophys. Res., 104, 30937-30946.

Baumgaertner A. J. G., Seppälä, A., Jöckel, P. and Clilverd, M. A., 2011. Geomagnetic activity related NOx enhancements and polar surface air temperature variability in a chemistry climate model: Modulation of the NAM index. Atmos. Chem. Phys., 11, 4521-31.

Boberg F. and Lundstedt, H., 2002. Solar wind variations related to fluctuations of the North Atlantic Oscillation. Geophys. Res. Lett., 29, 1718.

Boberg F. and Lundstedt, H., 2003. Solar wind electric field modulation of the NAO: a correlation analysis in the lower atmosphere. Geophys. Res. Lett., 30, 1825.

Ermolli, I, Matthes, K., Dudok, W. T., Krivova, N. A., Tourpali, K., Weber, M. and Woods, T. N., 2013. Recent variability of the solar spectral irradiance and its impact on climate modelling. Atmos. Chem. Phys., 13, 3945-77.

Finch, I. and Lockwood, M., 2007. Solar wind-magnetosphere coupling functions on timescales of 1 day to 1 year. Ann. Geophys., 25, 495-506. 
Graf, H-F., and Walter, K., 2005. Polar vortex controls coupling of North Atlantic Ocean and Atmosphere, Geophys. Res. Lett., 32, L01704, doi:10.1029/2004GL020664.

Gray, L.J., Beer, J., Geller, M., Haigh, J.D., Lockwood, M., Matthes, K., Cubasch, U., Fleitmann, D., Harrison, G., Hood, L., Luterbacher, J., Meehl, G.A., Shindell, D., van Geel, B., White, W., 2010. Solar influence on climate. Rev. Geophys. 48, RG4001, doi:10.1029/2009RG000282.

Haigh, J. D., 1996. The influence of solar variability on climate. Science, 272, 981-984.

Hoyt, D. V., and Schatten, K. H., 1997. The Role of the Sun in Climate Change, University Press, Oxford, UK.

Hurrell, J.W., Kushnir, Y., Ottersen, G., Visbeck, M., 2003. An overview of the North Atlantic oscillation, in: The North Atlantic Oscillation, Climate Significance and Environmental Impact. Geophysical Monograph 134, American Geophysical Union, Washington, DC.

Huth, R., Pokorná, L., Bochní cek, J., and Hejda, P., 2006. Solar cycle effects on modes of low-frequency circulation variability. J. Geophys. Res., 111, D22107, doi:10.1029/2005JD006813.

Huth, R., Pokorná, L., Bochní cek, J., and Hejda, P., 2009. Combined solar and QBO effects on the modes of low-frequency atmospheric variability in the Northern Hemisphere. J. Atmos. Solar-Terr. Phys., 71, 1471-1483.

King, J. H. and Papitashvili, N. E., 2005. Solar wind spatial scales in and 
comparisons of hourly Wind and ACE plasma and magnetic field data. J. Geophys. Res., 110, 1978-2012.

Kirkland, M.W., Tinsley, B.A., Hoeksema, J.T., 1996. Are stratospheric aerosols the missing link between tropospheric vorticity and Earth transits of the heliospheric current sheet? J. Geophys. Res. 101, D23, 29689-29699.

Kodera, K., 2002. Solar modulation of the North Atlantic Oscillation: implication in the spatial structure of the NAO. Geophys. Res. Lett., 29, 1218, doi10.1029/2001GL014557.

Kniveton, D. R. and Tinsley, B. A., 2004. Daily changes in global cloud cover and Earth transits of the heliospheric current sheet. J. Geophys. Res., 109, D11201.

Labitzke, K., 1987. Sunspots, the QBO, and the stratospheric temperature in the north pole region. Geophys. Res. Lett., 14, 535-537.

Labitzke, K. and van Loon, H., 1988. Associations between the 11-year solar cycle, the QBO, and the atmosphere, 1. The troposphere and the stratosphere of the northern hemisphere in winter. J. Atmos. Terr. Phys., 40, 197-206.

Lam, M. M., and Tinsley, B. A., (2016). Solar wind - atmospheric electricity cloud microphysics connections to weather and climate, J. Atmos. Solar Terr. Phys. In press, 2016.

Lam, M. M., Chisham, G. and Freeman, M. P., 2013. The interplanetary field influences mid-latitude surface atmospheric pressure. Env. Res. Lett., 8, 045001. 
Lam, M. M., Chisham, G. and Freeman, M. P., 2014. Solar wind - driven geopotential height anomalies originate in the Antarctic lower troposphere. Geophys. Res. Lett., 41, 6509-6514.

Li, X., D. N. Baker, Kanekal, S. G., Looper, M. and Temerin, M., 2001a. Long term measurements of radiation belts by SAMPEX and their variations. Geophys. Res. Lett., 28, 3827-30.

Li, X., Temerin, M., Baker, D. N., Reeves, G. D. and Larsen, D., $2001 b$. Quantitative prediction of radiation belt electrons at geostationary orbit based on solar wind measurements. Geophys. Res. Lett., 28, 1887-90.

Lu, H., Franzke, C., Martius, O., Jarvis, M. J., and Phillips, T., 2013. Solar wind dynamic pressure effect on planetary wave propagation and synoptic-scale Rossby wave breaking. J. Geophys. Res., 118, 4476-4493, doi:10.1002/jgrd.50374.

Maliniemi, V., Asikainen, T., Mursula, K. and Seppälä, A., 2013. QBO-dependent relation between electron precipitation and wintertime surface temperature. J. Geophys. Res., 118, 6302-10.

Markson, R. and Muir, M., 1980. Solar wind control of the Earth's electric field. Science, 208, 979-90.

Matthes K., Kuroda, Y., Kodera, K. and Langematz, U., 2006. Transfer of the solar signal from the stratosphere to the troposphere: Northern winter. J. Geophys. Res., 111, 1984-2012.

Mironova, I. A. and Usoskin, I. G., 2013. Possible effect of extreme solar 
energetic particle events of September-October 1989 on polar stratospheric aerosols: a case study. Atmos. Chem. Phys., 13, 8543-8550.

Mironova, I. A., Usoskin, I. G., 2014. Possible effect of strong solar energetic particle events on polar stratospheric aerosol: a summary of observational results. Environ. Res. Lett., 9, 015002.

Mironova, I. A., Usoskin, I. G., Kovaltsov, G. A. and Petelina, S. V., 2012 a. Possible effect of extreme solar energetic particle event of 20 January 2005 on polar stratospheric aerosols: direct observational evidence. Atmos. Chem. Phys., 12, 769-778.

Mironova, I., Tinsley, B. A. and Zhou, L. 2012b. The links between atmospheric vorticity, radiation belt electrons, and the solar wind. Adv. Space Res., 50, 783-90.

Perlwitz, J., and Graf, H-F., 2001. Troposphere-stratosphere dynamic coupling under strong and weak polar vortex conditions, Geophys. Res. Lett., 28, $271-274$.

Rayner, N. A., Parker, D. E., Horton, E. B., Folland, C. K., Alexander, L. V., Rowell, D. P., Kent, E. C., Kaplan, A., 2003. Global analyses of sea surface temperature, sea ice, and night marine air temperature since the late nineteenth century. J. Geophys. Res. $108 \quad$ (D14), 4407. 10.1029/2002JD002670

Reeves, G. D., Morley, S. K., Friedel, R. H. W., Henderson, M. G., Cayton, T. E., Cunningham, G., Blake, J. B., Christensen, R. A. and Thomsen D., 2011. On 
the relationship between relativistic electron flux and solar wind velocity: Paulikas and Blake revisited. J. Geophys. Res., 116, A02213.

Reichler, T., Kim, J., Manzini, E. and Kröger, J., 2012. A stratospheric connection to Atlantic climate variability. Nature Geoscience, 5, 783-787. doi:10.1038/ngeo1586.

Rozanov, E., Callis, L., Schlesinger, M., Yang, F., Andronova, N., and Zubov, V., 2005. Atmospheric response to NOy source due to energetic electron precipitation. Geophys. Res. Lett., 32, L14811, doi:10.1029/2005GL023041.

Rozanov, E., Calisto, M., Egorova, T., Peter, T. and Schmutz, W., 2012. Influence of the precipitating energetic particles on atmospheric chemistry and climate. Surv. Geophys., 33, 483-501.

Seppälä, A., Randall, C. E., Clilverd, M. A., Rozanov, E., and Rodger, C. J., 2009. Geomagnetic activity and polar surface air temperature variability. J. Geophys. Res., 114, A10312.

Seppälä, A. and Clilverd, M. A., 2014. Energetic particle forcing of the Northern Hemisphere winter stratosphere: Comparison to solar irradiance forcing. Front. Physiol., 2, 25.

Svensmark, H. and Friis-Christensen, E., 1997. Variation of cosmic rays fluxes and global cloud coverage-a missing link in solar-climate relationship. J. Atmos. Terr. Phys., 59, 1225-32.

Thejll, P., Christiansen, B., and Gleisner, H., 2003. On correlations between the North Atlantic Oscillation, geopotential heights, and geomagnetic activity. 
Geophys. Res. Lett., 30(6), 1374. doi:10.1029/2002GL016598.

Tinsley, B. A., 1996. Correlations of atmospheric dynamics with solar wind-induced changes of air - Earth current density into cloud tops. J. Geophys. Res., 101, 701-29.

Tinsley, B. A., 2008. The global atmospheric electric circuit and its effects on cloud microphysics. Rep. Prog. Phys., 71, 066801.

Tinsley, B. A., 2012. A working hypothesis for connections between electrically-induced changes in cloud microphysics and storm vorticity, with possible effects on circulation. Adv. Space Res., 50, 791-805.

Tinsley, B. A. and Deen, G. W., 1991. Apparent tropospheric response to MeV-GeV particle flux variations: A connection via electrofreezing of supercooled water in high-level clouds? J. Geophys. Res., 96, 22283-96.

Tinsley, B. A. and Heelis, R. A., 1993. Correlations of atmospheric dynamics with solar activity evidence for a connection via the solar wind, atmospheric electricity, and cloud microphysics. J. Geophys. Res., 98, 10375-84.

Torrence, C. and Compo, G. P., 1998. A Practical Guide to Wavelet Analysis. Bull. Amer. Meteor. Soc., 79, 61-78.

Veretenenko, S. and P. Thejll, P., 2004. Effects of energetic solar proton events on the cyclone development in the North Atlantic. J. Atmos. Solar-Terr. Phys., $66,393-405$.

Voiculescu, M., Usoskin, I. and Condurache, B. S., 2013. Clouds blown by the solar wind. Environ. Res. Lett., 8, 045032. 
Visbeck, M. H., Hurrell, J. W., Polvani, L., Polvani, L. and Cullen, H. M., 2001. The North Atlantic Oscillation: past, present, and future. Proceedings of the National Academy of Sciences, 98, 12876- 77.

Weimer, D. R., 1996. A flexible, IMF dependent model of high latitude electric potentials having "space weather" applications. Geophys. Res., Lett., 23, 2549-2552.

Zhou, L., Tinsley, B. A. and Huang, J., 2014. Effects on winter circulation of short and long term solar wind changes. Adv. Space Res., 54(12), 2478-90.

\section{Captions:}

Figure 1 . Time series analysis of monthly average of $10.7 \mathrm{~cm}$ solar radiation flux, solar wind electric field and solar wind speed, Fig 1(a), and the NAO index, Fig 1(b), as standardized monthly indices from 1963 to 2010. In Fig 1(a) the dashed line is for $10.7 \mathrm{~cm}$ solar flux, the solid line for solar wind electric field, and the dotted line for solar wind speed. In Fig. 1(c) the corresponding normalized power spectrum results are shown; the dashed lines represent the $95 \%$ confidence levels.

Figure 2. Seasonal mean spatial distribution of the correlation between SWS and SST; for MAM (a), JJA (b), SON (c), and DJF (d). The solid dark lines are the boundaries of the regions where the statistical confidence exceeds $99 \%$ with the t-test and the dashed dark lines are the boundaries of the regions where the statistical confidence exceeds $95 \%$. 
Figure 3. The regression of SST on SWS at locations near the peaks of the correlations in Fig. 2(d) for high (Fig. 3a) and low (Fig. 3b) latitudes.

Figure 4. As for figure 2(d), but for spatial distribution of the correlation between the SST and GEF as defined by Boberg and Lundstedt (2002) (a), and between SST and the $10.7 \mathrm{~cm}$ solar flux (b).

Figure 5. As for figure 2(d), but dividing winters into those with SWS above the mean value $(442.28 \mathrm{~km} / \mathrm{s})(\mathrm{a})$, and those below the average (b), to show the non-linearity of the response

Figure 6. As for figure 2(d), but dividing winters into those with average negative $B_{z}$ (a), and those with average positive $B_{z}$ (b).

Figure 7. As for figure 2(d), but dividing the DJF results into east (a) and west (b) QBO phase times. 

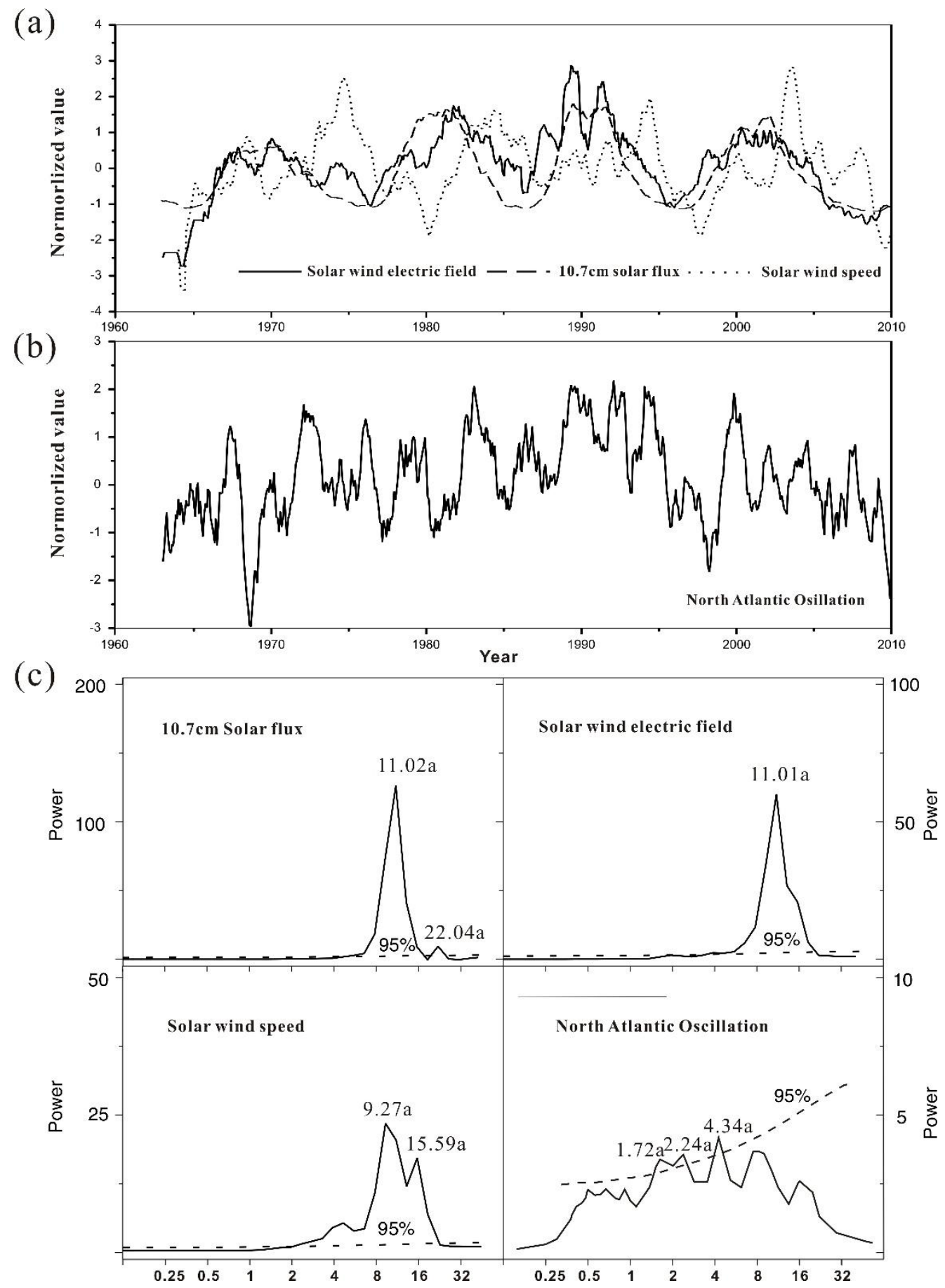

Figure 1 . Time series analysis of monthly average of $10.7 \mathrm{~cm}$ solar radiation flux, solar wind electric field and solar wind speed, Fig 1(a), and the NAO index, Fig 1(b), as standardized monthly indices from 1963 to 2010. In Fig 1(a) the dashed line is for $10.7 \mathrm{~cm}$ solar flux, the solid line for solar wind electric field, and the dotted line for solar wind speed. In Fig. 1(c) the corresponding normalized power spectrum results are shown; the dashed lines represent the $95 \%$ confidence levels. 

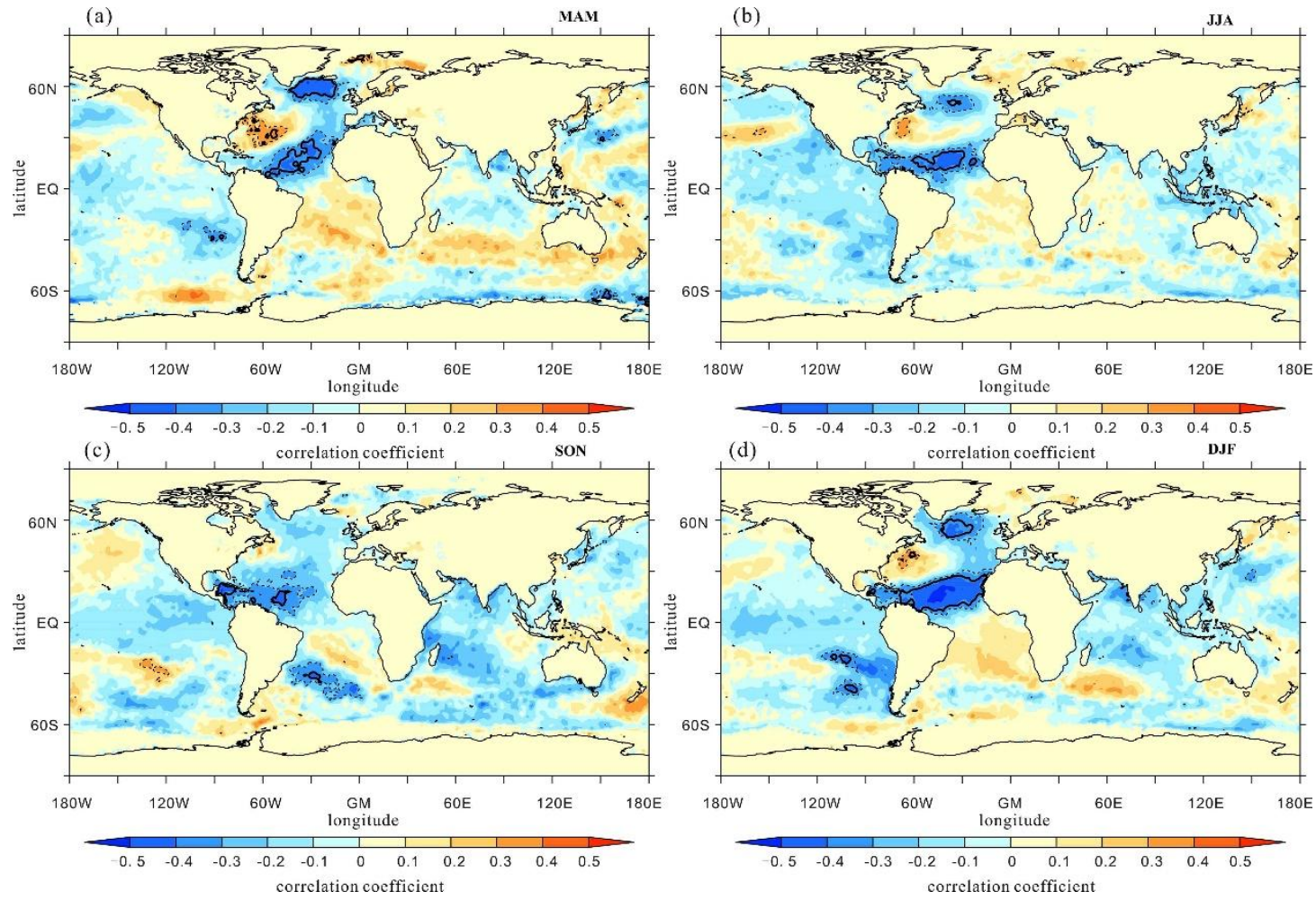

Figure 2. Seasonal mean spatial distribution of the correlation between SWS and SST; for MAM (a), JJA (b), SON (c), and DJF (d). The solid dark lines are the boundaries of the regions where the statistical confidence exceeds $99 \%$ with the t-test and the dashed dark lines are the boundaries of the regions where the statistical confidence exceeds $95 \%$. 

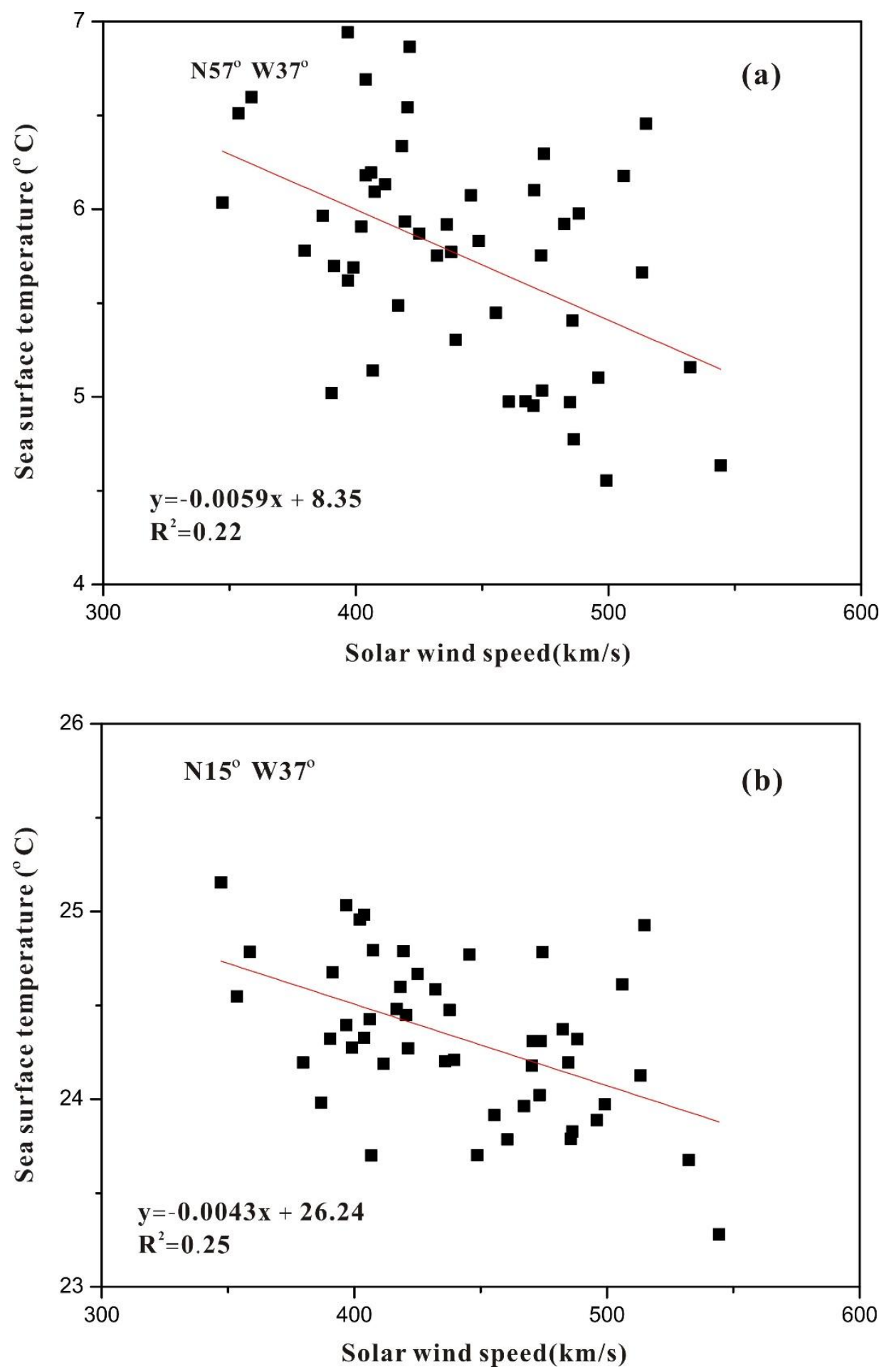

Figure 3. The regression of SST on SWS at locations near the peaks of the correlations in Fig. 2(d) for high (Fig. 3a) and low (Fig. 3b) latitudes. 

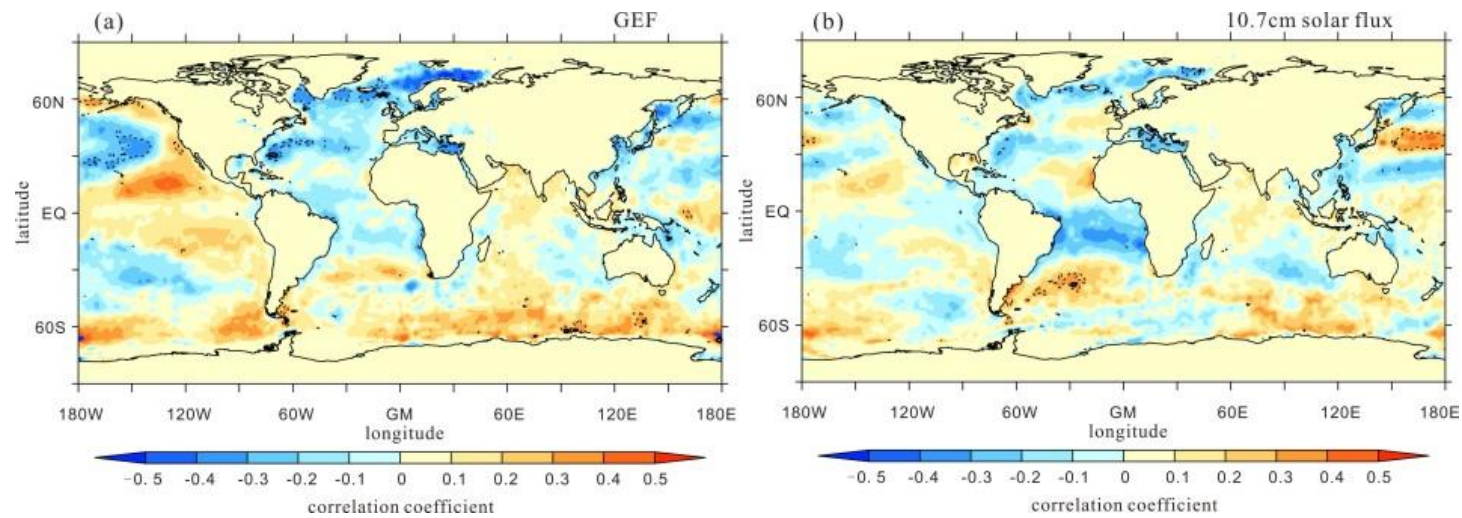

Figure 4. As for figure 2(d), but for spatial distribution of the correlation between the SST and GEF as defined by Boberg and Lundstedt (2002) (a), and between SST and the $10.7 \mathrm{~cm}$ solar flux (b).
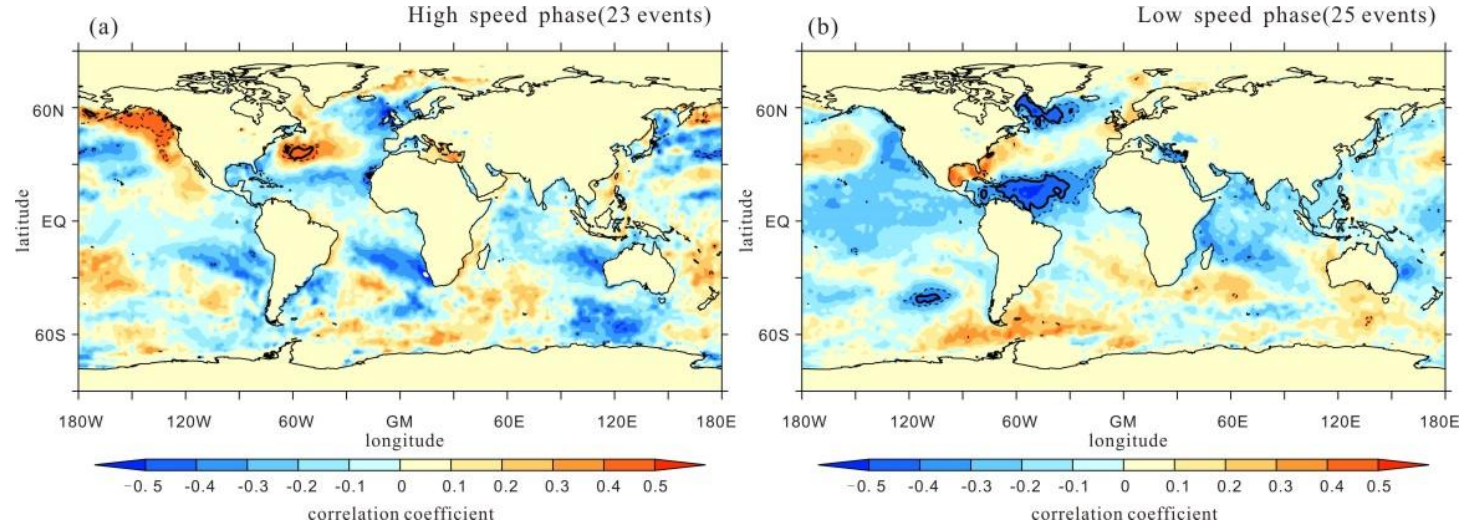

Figure 5. As for figure 2(d), but dividing winters into those with SWS above the mean value $(442.28 \mathrm{~km} / \mathrm{s})(\mathrm{a})$, and those below the average (b), to show the non-linearity of the response 

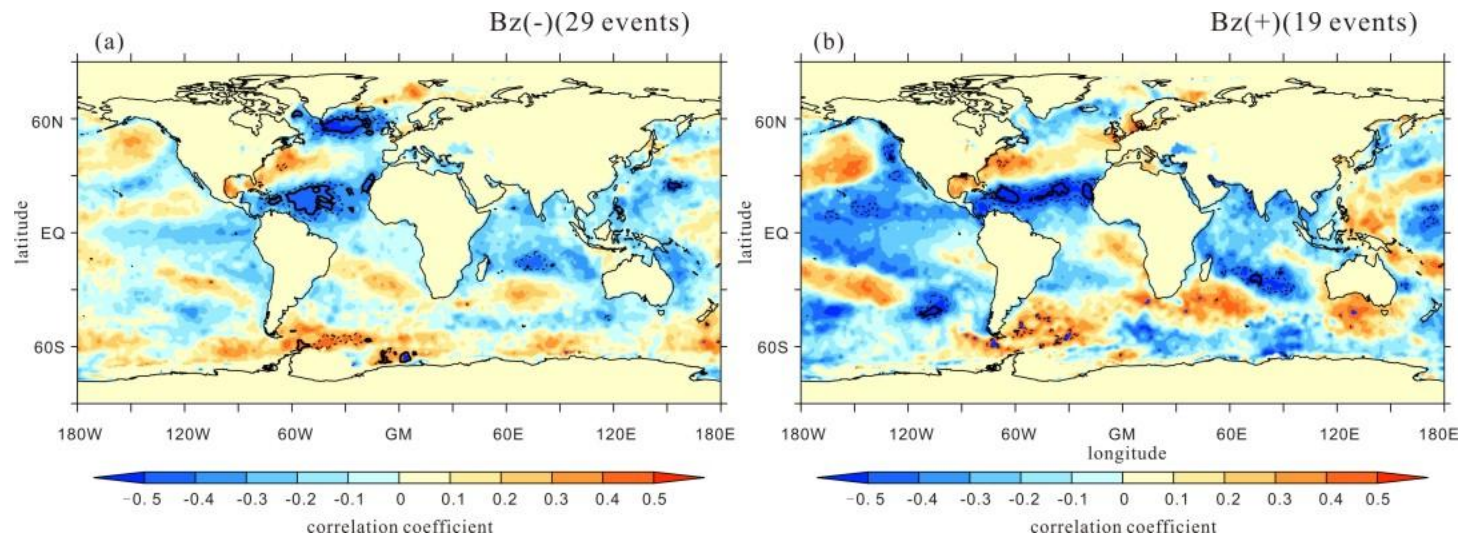

Figure 6. As for figure 2(d), but dividing winters into those with average negative $B_{z}$

(a), and those with average positive $B_{z}$ (b).
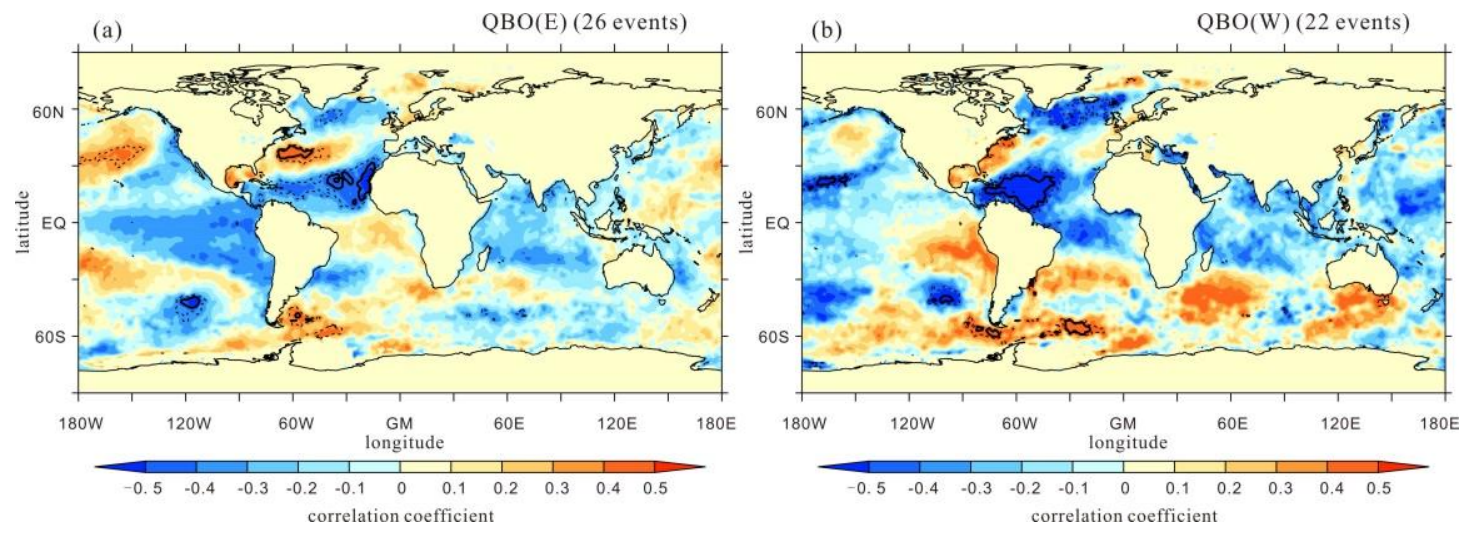

Figure 7. As for figure 2(d), but dividing the DJF results into east (a) and west (b) QBO phase times. 